\title{
Modelos para estimativa das propriedades mecânicas de compressão e tração na direção paralela às fibras
}

\author{
Models for estimation of mechanical properties of \\ compressive and tensile strength in the parallel direction \\ to the grains
}

\begin{abstract}
Anderson Renato Vobornik Wolenski
Fabrício Moura Dias

Rodrigo Guerra Peixoto

André Luís Christoforo

Francisco Antonio Rocco Lahr

\section{Resumo}

${ }^{1}$ Anderson Renato Vobornik

Wolenski

${ }^{1}$ Instituto Federal de Santa Catarina São Carlos - SC - Brasil

${ }^{2}$ Fabrício Moura Dias ${ }^{2}$ Centro Universitário do Leste de Minas Gerais Coronel Fabriciano - MG - Brasil

${ }^{3}$ Rodrigo Guerra Peixoto ${ }^{3}$ Universidade Federal de Minas

Belo Horizonte - MG - Brasil

${ }^{4}$ André Luís Christoforo 4Universidade Federal de São Carlos São Carlos - SP - Brasil

${ }^{5}$ Francisco Antonio Rocco Lahr 5Universidade de São Paulo São Carlos - SP - Brasil

Recebido em 31/10/18 Aceito em 07/09/19

A

s resistências mecânicas da madeira são calculadas, por questões de segurança, com base no seu valor característico. A norma brasileira para estruturas de madeira estabelece relações para estimar a resistência característica juntamente com três formas de caracterização da madeira, com destaque ao procedimento simplificado que permite, para espécies usuais, a obtenção dos valores característicos por meio de equações que correlacionam diferentes propriedades mecânicas. Ao considerar os resultados das resistências $\left(f_{\mathrm{c} 0} ; f_{\mathrm{t} 0}\right)$ e dos módulos de elasticidade $\left(\mathrm{E}_{\mathrm{c} 0} ; \mathrm{E}_{\mathrm{t} 0}\right)$ na compressão e tração paralelas às fibras de 40 espécies folhosas (960 experimentações com teor de umidade de $\approx 12 \%$ ), a precisão das relações $f_{\mathrm{c} 0, \mathrm{k}}=0,77 \cdot f_{\mathrm{t} 0, \mathrm{k}}$ e $\mathrm{E}_{\mathrm{c} 0}=\mathrm{E}_{\mathrm{t} 0}$ propostas pela norma foram avaliadas com base na análise de variância (ANOVA). Além do objetivo de avaliar tais equações, modelos de regressão linear, exponencial, logarítmico e geométrico foram adotados como proposta alternativa na estimativa de tais propriedades. As análises estatísticas validaram as equações propostas pela norma brasileira e a equação geométrica, proposta neste trabalho, mostrou-se como o modelo de melhor ajuste.

Palavras-chave: Madeiras folhosas. Modelos de regressão. Valores característicos.

\section{Abstract}

The mechanical strengths of wood are calculated, for safety reasons, based on their characteristic value. The Brazilian standard for timber structures establishes relationships to estimative the characteristic strength together with three forms of wood characterisation, with emphasis on the simplified procedure that allows, for usual species, obtaining the characteristic values through equations that correlate different mechanical properties. Considering the results of strengths $\left(f_{c 0} ; f_{t 0}\right)$ and of the modulus of elasticity $\left(E_{c 0} ; E_{t 0}\right)$ on the compressive and tensile parallel to the grains of 40 hardwood species (960 experimental determinations with $\approx 12 \%$ moisture content), the precision of the relations $f_{c 0, k}=0,77 \cdot f_{t 0, k}$ and $E_{c 0}=E_{t 0}$ proposed by the standard were evaluated based on the analysis of variance (ANOVA). Besides the objective of evaluating such equations, linear, exponential, logarithmic and geometric regression models were adopted as an alternative proposal in the estimation of such properties. The statistical analyzes validated the equations proposed by the Brazilian standard, and the geometric equation, proposed in this work, proved to be the best fit model.

Keywords: Hardwoods. Regression models. Characteristic values.
\end{abstract}




\section{Introdução}

A Norma Brasileira Regulamentadora - NBR 7190, Associação Brasileira de Normas Técnicas - ABNT (1997), discorre sobre projetos de estruturas de madeira, regulando seu uso na construção civil. Dentre os principais pontos estabelece os condicionantes para elaboração de projetos e as exigências para execução e controle de estruturas de madeira. Está pautada em métodos probabilísticos, como na verificação do estado limite último, que avalia a capacidade resistente à ruptura e a instabilidade da estrutura, e na verificação do estado limite de serviço, responsável por avaliar a deformação excessiva e a durabilidade da estrutura.

Em adição, a norma também traz os quesitos sobre arranjo estrutural, ações atuantes, modelo e análise estrutural, critérios relativos ao dimensionamento e detalhamento das estruturas e elementos de ligação, além de elencar métodos específicos para a obtenção das propriedades físicas e mecânicas das madeiras, prevendo para tanto métodos de caracterização completa, mínima ou simplificada, imprescindíveis para o dimensionamento estrutural em madeiras. Tal caracterização, segundo Christoforo et al. (2017), é justificada pela estrutura anatômica intrínseca a cada espécie, e que leva em consideração o tipo de esforço e a direção das fibras, assim como o teor de umidade da madeira.

Notadamente, uma caracterização completa de uma espécie é custosa, sendo poucos os laboratórios brasileiros capacitados para realizá-la. Tal questão fica evidenciada tendo em vista o grande número de experimentos exigidos por parte da NBR 7190 (ABNT, 1997, p. 15, item 6.3.1), sendo estes: ensaios de densidades básica $\left(\rho_{\text {bas }}\right)$ e aparente $\left(\rho_{\text {ap }}\right)$, resistências na compressão $\left(f_{\mathrm{c} 0}\right)$, na tração $\left(f_{\text {to }}\right)$, no cisalhamento $\left(f_{\mathrm{v} 0}\right)$ e no embutimento $\left(f_{\mathrm{e} 0}\right)$ paralelos às fibras e compressão $\left(f_{\mathrm{c} 90}\right)$, tração $\left(f_{\mathrm{t} 90}\right)$ e embutimento $\left(f_{\mathrm{e} 90}\right)$ normais às fibras, cabendo ressaltar que a normatização dos ensaios para determinação das propriedades é apresentada no Anexo B dessa norma.

Contudo, tal complexidade de ensaios pode ser minimizada, segundo a própria norma, ao detalhar a caracterização simplificada como sendo aquela realizada para espécies usuais ausentes de ensaios experimentais, exigindo para tanto resultados da $f_{\mathrm{co}}$ para um número mínimo de seis corpos de prova, além de propor a adoção de algumas relações que visam facilitar a obtenção das propriedades mecânicas (ABNT, 1997, p. 15, item 6.3.3), com destaque aos equacionamentos que preveem a correlação das resistências características à compressão $\left(f_{\mathrm{c} 0, \mathrm{k}}\right)$ e à tração $\left(f_{\mathrm{t} 0, \mathrm{k}}\right)\left[f_{\mathrm{c} 0, \mathrm{k}}=0,77 \cdot f_{\mathrm{t} 0, \mathrm{k}}\right]$, e a relação entre os módulos de elasticidade na compressão $\left(\mathrm{E}_{\mathrm{c} 0}\right)$ e na tração $\left(\mathrm{E}_{\mathrm{t} 0}\right)\left[\mathrm{E}_{\mathrm{c} 0}=\mathrm{E}_{\mathrm{t} 0}\right]$, ambos na direção paralela às fibras da madeira, sendo essas equações o objetivo central do estudo aqui apresentado.

Para tanto, este trabalho conduziu análises para 40 espécies de madeira do grupo das folhosas. Quando comparado com a NBR 7190 (ABNT, 1997, p. 90, Anexo E), traz valores médios para propriedades físicomecânicas de 43 espécies de madeiras nativas e de reflorestamento, conjunto este muito próximo às espécies aqui avaliadas, o que reforça o grau de confiabilidade das análises estatísticas aqui conduzidas exclusivamente para madeiras de florestas nativas.

Destaca-se, por fim, que em todos os métodos de caracterização a investigação direta de lotes de madeira serrada considerados homogêneos não deve ser composta por volumes superiores a $12 \mathrm{~m}^{3}$ (ABNT, 1997, Anexo B). A norma determina ainda que para lotes de madeira caracterizados pelos referidos métodos e com umidade contida no intervalo entre $10 \%$ e $20 \%$, a correção para a umidade padrão é de $12 \%$ (classe 1).

Tais exigências foram conduzidas para todas as propriedades avaliadas neste trabalho, com o objetivo de obter relações simplificadas capazes de fornecer propriedades mecânicas seguras para o prédimensionamento de estruturas de madeira.

\section{Referencial teórico}

Ao considerar a importância das propriedades mecânicas no dimensionamento de estruturas de madeira, como pontes, galpões e residências (RUELLE et al., 2011; DADZIE; AMOAH, 2015), torna-se relevante a avaliação de equações que objetivem estimar as propriedades mecânicas para espécies de uso estrutural, assim como prediz a NBR 7190 (ABNT, 1997, p. 15) ao citar o método simplificado com relações para obtenção de diferentes propriedades de resistência e rigidez para espécies usuais na ausência de resultados experimentais.

Conforme abordado por Logsdon et al. (2010), a NBR 7190 (ABNT, 1997) adota valores e critérios que podem não ser regra única quanto à obtenção dos valores característicos para as distintas solicitações de resistência. No trabalho em questão, os autores buscaram aferir o modelo para estimar a resistência $f_{\mathrm{co}, \mathrm{k}}$, com objetivo de obter um modelo estatístico mais adequado para tal solicitação. Para tanto, 100 corpos de

264 Wolenski, A. R. V.; Dias, F. M.; Peixoto, R. G.; Christoforo, A. L.; Lahr, F. A. R. 
prova da espécie angelim-pedra foram experimentalmente ensaiados para obtenção da referida resistência. Os autores concluíram que as equações propostas na referida norma são mais conservadores (a favor da segurança) por fornecerem valores menores para a $f_{\mathrm{c} 0, \mathrm{k}}$.

De modo congruente ao estudo anterior, Matos e Molina (2016) estudaram a correlação entre as resistências à compressão e ao cisalhamento $\left(f_{\mathrm{V} 0, \mathrm{k}} / f_{\mathrm{co}, \mathrm{k}}\right)$ das espécies de madeira de Pinus elliotti e Eucalyptus citriodora (Corymbia citriodora). Para tanto, os autores comparam a relação obtida experimentalmente com as constantes na NBR 7190 (ABNT, 1997) e na International Organization for Standardization - ISO 13910 (INTERNATIONAL..., 2005). A conclusão foi que as relações obtidas para ambas as espécies pela norma brasileira foram superiores às da europeia.

Enquanto os estudos anteriores se restringiram à análise de até duas espécies, a proposta de Dias e Lahr (2004) reporta à caracterização de 40 espécies de madeira nativas, visando estimar as propriedades físicomecânicas em função da densidade aparente $\left(\rho_{\text {ap }}\right)$, justificada pela inexistência de relações como esta na NBR 7190 (ABNT, 1997). Ressalta-se que na pesquisa de Dias e Lahr (2004) são avaliadas diferentes relações entre propriedades físicas e mecânicas; contudo, não aborda as resistências características, assim como proposto neste trabalho. Destaca-se ainda que dentre o conjunto de espécies estudadas no referido trabalho 32 espécies também são aqui avaliadas.

Outros autores, por sua vez, também avaliaram algumas das espécies aqui estudadas; entretanto, restringiram seus estudos às propriedades físicas de algumas madeiras como jatobá (Hymenea courbaril) (CAVALHEIRO et al., 2016; TIAGO et al., 2018); mandioqueira (Qualea paraensis) (ALMEIDA et al., 2017); itaúba (Mezilaurus itauba) (DA SILVA et al., 2014); garapa (Apuleia leiocarpa) (SORIANO et al., 2015); cedro-amargo (Cedrela odorata) (FERNANDES et al., 2018).

\section{Materiais e métodos}

Os valores das resistências características, para as 40 espécies avaliadas neste trabalho, na compressão e na tração paralela às fibras $\left(f_{\mathrm{c} 0, \mathrm{k}}\right.$ e $\left.f_{\mathrm{t} 0, \mathrm{k}}\right)$ e da rigidez para os mesmos esforços $\left(\mathrm{E}_{\mathrm{c} 0}\right.$ e $\left.\mathrm{E}_{\mathrm{t} 0}\right)$ foram obtidos seguindo as premissas e os métodos de ensaio e de cálculo da NBR 7190 (ABNT, 1997, p. 47, Anexo B). Os equacionamentos empregados para avaliar tais propriedades são sumarizados na sequência.

Além disso, são detalhados os modelos de regressão empregados como estimador das propriedades de resistência e rigidez, assim como da análise de variância (ANOVA) para avaliação da equivalência das equações $f_{\mathrm{c} 0, \mathrm{k}}=0,77 \cdot f_{\mathrm{t} 0, \mathrm{k}}$ e $\mathrm{E}_{\mathrm{c} 0}=\mathrm{E}_{\mathrm{t} 0}$ ao considerar o conjunto de resultados das 40 espécies de madeira do grupo das folhosas.

Os ensaios foram realizados nas dependências do Laboratório de Madeiras e de Estruturas de Madeira (LaMEM), Departamento de Engenharia de Estruturas, Escola de Engenharia de São Carlos, Universidade de São Paulo. Para cada uma das propriedades de resistência $\left(f_{\mathrm{c} 0}\right.$ e $\left.f_{\mathrm{t} 0}\right)$ e de rigidez $\left(\mathrm{E}_{\mathrm{c} 0}\right.$ e $\left.\mathrm{E}_{\mathrm{t} 0}\right)$, ensaiaram-se 12 corpos de prova para cada uma das 40 espécies (Tabela 1), o que resultou em um total de $2 \times 12 \times 40=$ 960 determinações experimentais.

\section{Valores característicos}

As propriedades mecânicas de resistência (Equação 1) e de rigidez (Equação 2) tiveram seus valores corrigidos para o teor de umidade de 12\%, assim como prediz a NBR 7190 (ABNT, 1997), sendo 12\% o teor de umidadade de equilíbrio estabelecido por esse documento.

$f_{12}=f_{\mathrm{U} \%}\left[1+\frac{3(\mathrm{U} \%-12)}{100}\right]$

$\mathrm{E}_{12}=\mathrm{E}_{\mathrm{U} \%}\left[1+\frac{2(\mathrm{U} \%-12)}{100}\right]$

Onde:

$f_{12}$ é a resistência corrigida para a umidade de $12 \%$;

$f_{\mathrm{U} \%}$ é a resistência para o teor de umidade U\%;

$\mathrm{E}_{12}$ é consiste no módulo de elasticidade corrigido para a umidade de $12 \%$; e

$\mathrm{E}_{\mathrm{U} \%}$ é o módulo para o teor de umidade U\%. 
Tabela 1 - Nomes e identificação (ID) das $\mathbf{4 0}$ espécies nativas do grupo das folhosas

\begin{tabular}{|c|c|c|c|c|c|}
\hline ID & Popular & Científico $^{1}$ & ID & Popular & Científico $^{1}$ \\
\hline 01 & Angelim-saia & Vatairea cf. guianensis & 21 & Guajará & Micropholis venulosa \\
\hline 02 & Angelim-vermelho & Dinizia excelsa & 22 & Guarucaia & Peltophorum dubium \\
\hline 03 & Angico-branco & Parkia cf. pendula & 23 & Itaúba & Mezilaurus itauba \\
\hline 04 & Angico-preto & Anadenanthera colubrina & 24 & Jatobá & Hymenaea courbaril \\
\hline 05 & Branquilho & $\underline{\text { Sebastiania commersoniana }}$ & 25 & Louro-preto & Ocotea neesiana \\
\hline 06 & Cafearana & Andira anthelmia & 26 & Louro-verde & Sextonia cf. rubra \\
\hline 07 & Cambará-rosa & Erisma cf. fuscum & 27 & Maçaranduba & Manilkara cf. inundata \\
\hline 08 & Canafístula & Cassia ferruginea & 28 & Mandioqueira & Qualea paraensis \\
\hline 09 & Castanheira & Bertholletia excelsa & 29 & Oiticica-amarela & Clarisia racemosa \\
\hline 10 & Castelo & Calycophyllum multiflorum & 30 & Oiuchu & Pradosia sp. \\
\hline 11 & Catanudo & Calophyllum longifolium & 31 & Parinari & $\underline{\text { Parinari excelsa }}$ \\
\hline 12 & Cedro-amargo & Cedrela odorata & 32 & Pau-óleo & Copaifera langsdorffii \\
\hline 13 & Cedro-doce & Cedrela cf. fissilis & 33 & Piolho & Tapirira sp. \\
\hline 14 & Cedrorana & Cedrelinga cateniformis & 34 & Quarubarana & Erisma uncinatum \\
\hline 15 & Champanhe & Dipteryx odorata & 35 & Quina-rosa & Geissospermum sericeum \\
\hline 16 & Copaíba & Copaifera multijuga & 36 & Rabo-de-arraia & $\underline{\text { Vochysia haenkeana }}$ \\
\hline 17 & Cutiúba & $\underline{\text { Goupia paraensis }}$ & 37 & Sucupira & Diplotropis sp. \\
\hline 18 & Garapa & Apuleia leiocarpa & 38 & Tachi & Tachigali glauca \\
\hline 19 & Goiabão & Planchonella pachycarpa & 39 & Tatajuba & Bagassa guianensis \\
\hline 20 & Guaiçara & Luetzelburgia cf. guaissara & 40 & Umirana & $\underline{\text { Ruizterania retusa }}$ \\
\hline
\end{tabular}

Nota: ${ }^{1}$ Flora do Brasil (2019).

Para a determinação dos valores característicos das resistências à compressão e à tração das espécies avaliadas, necessárias para compor a resistência de cálculo para uma determinada solicitação em um eventual projeto estrutural, devem-se considerar os valores das resistências às solicitações mecânicas das madeiras obtidos a partir do seu valor característico $\left(f_{\mathrm{w}, \mathrm{k}}\right)$, que corresponde a um percentil de $5 \%$ da distribuição de resistências, e expresso segundo a Equação 3, sendo $f_{m}$ o valor médio da resistência e $\delta$ o respectivo desvio padrão.

$f_{\mathrm{w}, \mathrm{k}}=f_{\mathrm{m}}[1-1,645 \delta]$

Eq. 3

Em adição, a NBR 7190 (ABNT, 1997, p. 18, item 6.4.7) exige que os projetos de estruturas de madeira sejam dimensionados sob a hipótese de linearidade cinemática (pequenos deslocamentos), além de exigir resistência à ação das forças atuantes. Assim, a metodologia probabilística da referida norma supõe a normalidade nas distribuições dos valores da resistência, a favor da segurança, considerando um coeficiente de variação $\delta=18 \%$ para as resistências à tração e compressão, tal como expressa a Equação 4.

$f_{\mathrm{w}, \mathrm{k}}=f_{\mathrm{m}}[1-1,645 \delta] \approx 0,70 f_{\mathrm{m}}$

Eq. 4

Por outro lado, para uma investigação direta da resistência, a normativa prevê a empregabilidade da Equação 5 , que estima o valor de $f_{w, k}$ na forma:

$f_{\mathrm{w}, \mathrm{k}}=\left[2 \frac{f_{1}+f_{2}+f_{3}+\cdots+f\left(\frac{\mathrm{n}}{2}-1\right)}{\left(\frac{\mathrm{n}}{2}-1\right)}-f_{\left(\frac{\mathrm{n}}{2}\right)}\right] \cdot 1,10$

Eq. 5

Em que $f_{n}$ corresponde a n resultados da resistência à determinada solicitação, devendo ser colocados em ordem crescente $\left(f_{1}<f_{2}<f_{3}<\cdots<f_{\mathrm{n}}\right)$ ao desconsiderar o maior valor se o número de corpos de prova for ímpar. Ressalta-se que tal equação baseia-se no estimador $z_{b}$ (Equação 6) para uma amostra de $2 \mathrm{~m}$ valores $x_{1}+x_{2}+x_{3}+\cdots+x_{2 m}$.

$z_{b}=\left[2 \frac{x_{1}+x_{2}+x_{3}+\cdots+x_{(m-1)}}{(m-1)}-x_{m}\right]$

Eq. 6

Contudo, para uma distribuição de extremos centrada no valor característico, a Equação 5 tem um acréscimo de $10 \%$, o que evita, segundo Logsdon et al. (2010), que 50\% das estimativas sejam realizadas por valores abaixo da resistência característica. A norma brasileira adota como valor de $f_{\mathrm{w}, \mathrm{k}} \mathrm{o}$ maior dos valores

266 Wolenski, A. R. V.; Dias, F. M.; Peixoto, R. G.; Christoforo, A. L.; Lahr, F. A. R. 
compreendidos entre a resistência $f_{1}$, a equivalente a $70 \%$ do valor de $f_{m}$ (Equação 4) obtido pela média das amostras ensaiadas, e o valor obtido pela Equação 5 (ABNT, 1997, p. 18, item 6.4.8).

Destaca-se que o valor característico da resistência $f_{\mathrm{c} 0, \mathrm{k}}$ é a medida responsável por enquadrar as diferentes espécies de madeira em quatro classes de resistência (CR) referentes ao grupo das folhosas (ABNT, 1997, p. 16, item 6.3.5). Portanto, tais resistências foram calculadas conforme a Equação 5 com representatividade em todas as classes da Tabela 2, o que justifica a aplicabilidade das 40 espécies avaliadas neste trabalho para uso estrutural.

\section{Relações entre as propriedades}

A NBR 7190 (ABNT, 1997, p. 15, item 6.3.3) possibilita uma caracterização simplificada da madeira com base nos resultados de compressão na direção paralela às fibras. Para tanto, prevê algumas relações, com destaque para a Equação 7, foco deste estudo e que prevê a obtenção das resistências características $f_{\text {c0,k }}$ em função da $f_{\mathrm{t} 0, \mathrm{k}}$ e vice-versa.

$f_{\mathrm{c} 0, \mathrm{k}}=0,77 \cdot f_{\mathrm{t} 0, \mathrm{k}}$

Deve-se ressaltar, contudo, que tal equação se restringe à caracterização de espécies usuais que, por ventura, tenham ausência de resultados experimentais advindos de uma caracterização completa. Da mesma forma, a NBR 7190 (ABNT, 1997, p. 16, item 6.3.4) também admite que o valor médio do módulo de elasticidade $\mathrm{E}_{\mathrm{c} 0}$ é equivalente ao valor do $\mathrm{E}_{\mathrm{t} 0}$, expresso na forma:

$\mathrm{E}_{\mathrm{c} 0}=\mathrm{E}_{\mathrm{t} 0}$

Eq. 8

Notadamente, a simplicidade exposta pelas equações lineares acima podem ou não serem coerentes com resultados reais advindos de análises experimentais. Nesse contexto, este trabalho busca responder tal indagação e mostrar, estatisticamente, a validade das Equações 7 e 8 a partir da análise do conjunto das 40 espécies (Tabela 1), de modo a considerar um espaço amostral significativo, fornecido pela variabilidade do conjunto de respostas dos ensaios experimentais dessas espécies.

\section{Análise estatística}

A análise de variância (ANOVA), teste $\mathrm{F}(\mathrm{p}<0,05)$, foi adotada para verificar a precisão das Equações 7 e 8 propostas pela norma brasileira. Da ANOVA, a hipótese nula consistiu na equivalência das médias das propriedades $\left(f_{\mathrm{c} 0, \mathrm{k}}\right.$ e $0,77 \cdot f_{\mathrm{t} 0, \mathrm{k}} ; \mathrm{E}_{\mathrm{c} 0}$ e $\left.\mathrm{E}_{\mathrm{t} 0}\right)$, e na não equivalência das médias dos grupos confrontados como hipótese alternativa. Pelas hipóteses admitidas, $\mathrm{p} \geq 0,05$ implica aceitar a hipótese nula (as médias dos grupos confrontados são estatisticamente equivalentes entre si), e de rejeitá-la no caso de p < 0,05 (grupos com médias significativamente diferentes).

A premissa de normalidade na distribuição dos resíduos da ANOVA é avaliada pelo teste de AndersonDarling (WEERAHANDI, 1995). Pela formulação, p > 0,05 implica aceitar a normalidade na distribuição dos resíduos, e de rejeitar essa hipótese em caso contrário.

Tabela 2 - Classes de resistência (CR) das folhosas, conforme a NBR 7190 (ABNT, 1997)

\begin{tabular}{c|c|c|c|c}
\hline CR & $\boldsymbol{f}_{\mathbf{c} 0, \mathbf{k}}(\mathbf{M P a})$ & $\boldsymbol{E}_{\mathrm{c} 0}(\mathbf{M P a})$ & $\boldsymbol{\rho}_{\text {bas }}\left(\mathbf{k g} / \mathbf{m}^{\mathbf{3}}\right)$ & $\boldsymbol{\rho}_{\text {ap }}\left(\mathbf{k g} / \mathbf{m}^{\mathbf{3}}\right)$ \\
\hline C20 & 20,00 & 9500 & 500,00 & 650,00 \\
C30 & 30,00 & 14500 & 650,00 & 800,00 \\
C40 & 40,00 & 19500 & 750,00 & 950,00 \\
C60 & 60,00 & 24500 & 800,00 & 1000,00 \\
\hline
\end{tabular}

Nota: $\boldsymbol{f}_{\mathbf{c} 0, \mathbf{k}}$ e $\boldsymbol{E}_{\mathbf{c} 0}$ - resistência característica e rigidez na compressão paralela às fibras; $\boldsymbol{\rho}_{\text {bas }}$ e $\boldsymbol{\rho}_{\mathrm{ap}}$ - densidades básica e aparente. 
Avaliadas a precisão das Equações 7 e 8, na sequência, modelos de regressão a dois parâmetros (a e b) avaliados via ANOVA (F; p < 0,05) foram utilizados para relacionar as variáveis das Equações 7 e 8 , consistindo em modelos alternativos frente aos propostos pela norma brasileira. Os modelos de regressão consistiram no linear (Equação 9), exponencial (Equação 10), logarítmico (Equação 11) e geométrico (Equação 12).

$\mathrm{Y}=\mathrm{a}+\mathrm{b} \cdot \mathrm{X}$

Eq. 9

$Y=a \cdot e^{b \cdot X}$

Eq. 10

$\mathrm{Y}=\mathrm{a}+\mathrm{b} \cdot \ln (\mathrm{X})$

Eq. 11

$\mathrm{Y}=\mathrm{a} \cdot \mathrm{X}^{\mathrm{b}}$

Eq. 12

Nas Equações de 9 a 12, Y é a variável dependente e X é a variável independente, e a e b consistem nos parâmetros dos modelos obtidos pelo método dos mínimos quadrados. Além do uso da ANOVA, que permite aceitar ou não a representatividade dos modelos de regressão testados, os valores do coeficiente de determinação ajustado $\left(\mathrm{R}_{\mathrm{aj}}^{2}\right)$ permitem eleger, dentre os modelos significativos, o de melhor ajuste.

\section{Resultados e discussão}

Nas Tabelas de 3 a 6 são apresentados os valores médios (em MPa), o desvio padrão (DP), o coeficiente de variação $(\mathrm{CV} \%)$ e o intervalo de confiança (IC) da média $(\mathrm{p}<0,05)$, para as propriedades mecânicas das 40 espécies de madeira folhosas avaliadas neste trabalho.

É importante destacar que dentre as 40 espécies avaliadas (Tabela 1) muitas também foram objetos de estudos em outras pesquisas. Pode-se então comparar as propriedades mecânicas $\left(f_{\mathrm{c} 0}, f_{\mathrm{t} 0}, \mathrm{E}_{\mathrm{c} 0}, \mathrm{E}_{\mathrm{t} 0}\right)$ apresentadas nas Tabelas 3, 4, 5 e 6 com seus respectivos intervalos de confiança (IC), de modo a validar os valores das propriedades aqui estudadas.

Tabela 3 - Resultados estatísticos obtidos da resistência média na compressão, $\boldsymbol{f}_{\mathrm{c} 0}$ (MPa)

\begin{tabular}{c|c|c|c|c|c|c|c|c|c}
\hline ID & $\boldsymbol{f}_{\mathbf{c 0}}$ & $\mathbf{D P}$ & $\mathbf{C V} \%$ & $\mathbf{I C}$ & $\mathbf{I D}$ & $\boldsymbol{f}_{\mathbf{c 0}}$ & $\mathbf{D P}$ & $\mathbf{C V} \%$ & IC \\
\hline 01 & 63,11 & 8,84 & 14,02 & $(58,46 ; 67,76)$ & 21 & 74,97 & 6,05 & 8,07 & $(70,33 ; 79,55)$ \\
\hline 02 & 77,53 & 5,85 & 7,54 & $(72,88 ; 82,18)$ & 22 & 62,38 & 5,67 & 9,09 & $(57,73 ; 67,03)$ \\
\hline 03 & 44,35 & 3,79 & 8,54 & $(39,70 ; 49,00)$ & 23 & 69,04 & 4,24 & 6,14 & $(64,39 ; 73,69)$ \\
\hline 04 & 72,52 & 14,33 & 19,76 & $(67,87 ; 77,17)$ & 24 & 93,27 & 7,79 & 8,35 & $(88,63 ; 97,92)$ \\
\hline 05 & 48,47 & 3,80 & 7,84 & $(43,83 ; 53,12)$ & 25 & 59,99 & 7,60 & 12,66 & $(55,34 ; 64,64)$ \\
\hline 06 & 57,58 & 12,13 & 21,06 & $(52,93 ; 62,23)$ & 26 & 52,95 & 5,67 & 10,71 & $(48,30 ; 57,60)$ \\
\hline 07 & 34,48 & 5,09 & 14,75 & $(29,83 ; 39,13)$ & 27 & 82,93 & 6,18 & 7,45 & $(78,28 ; 87,57)$ \\
\hline 08 & 51,96 & 9,81 & 18,88 & $(47,31 ; 56,61)$ & 28 & 69,17 & 11,09 & 16,04 & $(64,52 ; 73,82)$ \\
\hline 09 & 48,17 & 6,69 & 13,89 & $(43,52 ; 52,82)$ & 29 & 70,33 & 6,86 & 9,76 & $(65,68 ; 74,97)$ \\
\hline 10 & 54,80 & 2,81 & 512 & $(50,15 ; 59,45)$ & 30 & 77,35 & 8,53 & 11,03 & $(72,70 ; 82,00)$ \\
\hline 11 & 50,58 & 3,34 & 6,61 & $(47,84 ; 53,31)$ & 31 & 60,56 & 5,06 & 8,35 & $(55,91 ; 65,21)$ \\
\hline 12 & 39,46 & 4,72 & 11,97 & $(34,81 ; 44,11)$ & 32 & 53,34 & 8,06 & 15,10 & $(48,69 ; 57,99)$ \\
\hline 13 & 31,48 & 5,63 & 17,88 & $(26,83 ; 36,12)$ & 33 & 61,88 & 13,34 & 21,55 & $(57,23 ; 66,53)$ \\
\hline 14 & 41,52 & 9,46 & 22,77 & $(36,87 ; 46,17)$ & 34 & 37,83 & 9,62 & 25,42 & $(33,18 ; 42,48)$ \\
\hline 15 & 93,16 & 5,07 & 5,45 & $(88,51 ; 97,81)$ & 35 & 59,17 & 2,62 & 4,43 & $(54,52 ; 63,82)$ \\
\hline 16 & 50,21 & 5,95 & 11,85 & $(45,56 ; 54,86)$ & 36 & 57,58 & 11,50 & 19,97 & $(52,93 ; 62,23)$ \\
\hline 17 & 78,97 & 15,52 & 19,65 & $(74,32 ; 83,62)$ & 37 & 94,53 & 7,17 & 7,59 & $(89,92 ; 99,15)$ \\
\hline 18 & 74,03 & 8,48 & 11,45 & $(69,38 ; 78,67)$ & 38 & 87,75 & 9,73 & 11,08 & $(83,10 ; 92,40)$ \\
\hline 19 & 48,46 & 5,98 & 12,34 & $(43,81 ; 53,11)$ & 39 & 80,16 & 14,88 & 18,56 & $(75,51 ; 84,81)$ \\
\hline 20 & 71,41 & 8,89 & 12,46 & $(66,76 ; 76,06)$ & 40 & 53,27 & 4,11 & 7,72 & $(48,62 ; 57,92)$ \\
\hline
\end{tabular}

Nota: $f_{\mathrm{c} 0}$ - resistência média na compressão; DP - desvio padrão; CV\% - coeficiente de variação; IC - intervalo de confiança.

268 Wolenski, A. R. V.; Dias, F. M.; Peixoto, R. G.; Christoforo, A. L.; Lahr, F. A. R. 
Tabela 4 - Resultados estatísticos obtidos da resistência média na tração, $\boldsymbol{f}_{\text {to }}$ (MPa)

\begin{tabular}{c|c|c|c|c|c|c|c|c|c}
\hline ID & $\boldsymbol{f}_{\mathbf{t} \mathbf{0}}$ & $\mathbf{D P}$ & $\mathbf{C V} \%$ & $\mathbf{I C}$ & ID & $\boldsymbol{f}_{\mathbf{t} \mathbf{0}}$ & $\mathbf{D P}$ & $\mathbf{C V} \%$ & $\mathbf{I C}$ \\
\hline 01 & 95,88 & 30,71 & 32,03 & $(81,53 ; 110,23)$ & 21 & 119,18 & 27,34 & 22,94 & $(105,41 ; 132,96)$ \\
\hline 02 & 104,90 & 34,58 & 32,96 & $(91,16 ; 118,64)$ & 22 & 74,88 & 12,92 & 17,26 & $(61,14 ; 88,61)$ \\
\hline 03 & 67,10 & 10,40 & 15,49 & $(53,37 ; 80,84)$ & 23 & 103,72 & 29,40 & 28,35 & $(89,98 ; 117,45)$ \\
\hline 04 & 109,5 & 34,2 & 31,20 & $(95,2 ; 123,8)$ & 24 & 165,27 & 32,56 & 19,70 & $(151,53 ; 179,00)$ \\
\hline 05 & 87,88 & 19,14 & 21,78 & $(74,14 ; 101,61)$ & 25 & 74,07 & 13,27 & 17,92 & $(60,33 ; 87,80)$ \\
\hline 06 & 80,42 & 23,85 & 29,66 & $(66,69 ; 94,16)$ & 26 & 82,80 & 10,40 & 12,55 & $(69,06 ; 96,54)$ \\
\hline 07 & 45,41 & 13,80 & 30,39 & $(31,67 ; 59,15)$ & 27 & 138,48 & 25,14 & 18,16 & $(124,74 ; 152,21)$ \\
\hline 08 & 84,92 & 21,52 & 25,34 & $(71,18 ; 98,65)$ & 28 & 93,04 & 30,23 & 32,49 & $(79,30 ; 106,78)$ \\
\hline 09 & 88,50 & 21,74 & 24,56 & $(74,76 ; 102,24)$ & 29 & 85,83 & 12,82 & 14,94 & $(72,09 ; 99,56)$ \\
\hline 10 & 103,64 & 15,11 & 14,58 & $(89,90 ; 117,38)$ & 30 & 121,8 & 35,6 & 29,25 & $(108,1 ; 135,6)$ \\
\hline 11 & 65,37 & 24,89 & 38,07 & $(53,94 ; 76,81)$ & 31 & 113,4 & 35,9 & 31,64 & $(99,6 ; 127,1)$ \\
\hline 12 & 58,20 & 14,50 & 24,91 & $(44,46 ; 71,94)$ & 32 & 69,29 & 17,40 & 25,11 & $(55,55 ; 83,03)$ \\
\hline 13 & 67,23 & 15,33 & 22,80 & $(53,50 ; 80,97)$ & 33 & 73,05 & 23,93 & 32,76 & $(59,31 ; 86,79)$ \\
\hline 14 & 61,85 & 16,63 & 26,89 & $(48,11 ; 75,59)$ & 34 & 58,05 & 13,11 & 22,58 & $(44,31 ; 71,79)$ \\
\hline 15 & 121,70 & 26,25 & 21,57 & $(107,35 ; 136,05)$ & 35 & 116,64 & 28,23 & 24,20 & $(102,29 ; 130,98)$ \\
\hline 16 & 71,13 & 16,44 & 23,11 & $(57,39 ; 84,86)$ & 36 & 70,94 & 19,51 & 27,49 & $(57,20 ; 84,68)$ \\
\hline 17 & 107,5 & 35,3 & 32,81 & $(93,7 ; 121,2)$ & 37 & 130,94 & 22,36 & 17,07 & $(117,17 ; 144,71)$ \\
\hline 18 & 107,64 & 24,22 & 22,50 & $(93,90 ; 121,38)$ & 38 & 106,75 & 27,45 & 25,71 & $(93,01 ; 120,49)$ \\
\hline 19 & 119,3 & 35,4 & 29,70 & $(105,6 ; 133,1)$ & 39 & 92,92 & 20,90 & 22,49 & $(79,19 ; 106,66)$ \\
\hline 20 & 115,13 & 23,32 & 20,25 & $(100,78 ; 129,48)$ & 40 & 57,13 & 17,48 & 30,60 & $(43,40 ; 70,87)$ \\
\hline
\end{tabular}

Nota: $f_{\mathrm{t} 0}$ - resistência média na tração; DP - desvio padrão; CV\% - coeficiente de variação; IC - intervalo de confiança.

Tabela 5 - Resultados estatísticos obtidos da rigidez na compressão, $\mathbf{E}_{\mathbf{c 0}}$ (MPa)

\begin{tabular}{c|c|c|c|c|c|c|c|c|c}
\hline ID & $\mathbf{E}_{\mathbf{c 0}}$ & $\mathbf{D P}$ & $\mathbf{C V} \%$ & $\mathbf{I C}$ & $\mathbf{I D}$ & $\mathbf{E}_{\mathbf{c} 0}$ & $\mathbf{D P}$ & $\mathbf{C V} \%$ & $\mathbf{I C}$ \\
\hline 01 & 19748 & 3256 & 16,49 & $(18228 ; 21268)$ & 21 & 14489 & 1612 & 10,13 & $(12967 ; 16012)$ \\
\hline 02 & 16695 & 2974 & 17,82 & $(15175 ; 18215)$ & 22 & 16214 & 2139 & 13,19 & $(14694 ; 17734)$ \\
\hline 03 & 14962 & 2886 & 19,29 & $(13442 ; 16482)$ & 23 & 17443 & 1785 & 10,23 & $(15923 ; 18963)$ \\
\hline 04 & 15375 & 5331 & 34,67 & $(13855 ; 16895)$ & 24 & 24741 & 3691 & 14,92 & $(23221 ; 26261)$ \\
\hline 05 & 13813 & 1956 & 14,16 & $(12293 ; 15333)$ & 25 & 15186 & 1505 & 9,91 & $(13666 ; 16706)$ \\
\hline 06 & 14089 & 3671 & 26,06 & $(12569 ; 15609)$ & 26 & 15050 & 3083 & 20,48 & $(13530 ; 16570)$ \\
\hline 07 & 12967 & 2335 & 18,00 & $(11447 ; 14487)$ & 27 & 21900 & 2680 & 12,24 & $(20380 ; 23420)$ \\
\hline 08 & 14613 & 2718 & 18,60 & $(13093 ; 16133)$ & 28 & 19498 & 2615 & 13,41 & $(17978 ; 21018)$ \\
\hline 09 & 13639 & 2723 & 19,96 & $(12119 ; 15159)$ & 29 & 14719 & 1362 & 9,25 & $(13199 ; 16239)$ \\
\hline 10 & 11188 & 1219 & 10,90 & $(9668 ; 12708)$ & 30 & 17718 & 4403 & 24,85 & $(16130 ; 19305)$ \\
\hline 11 & 13029 & 2306 & 17,70 & $(12076 ; 13983)$ & 31 & 22088 & 2755 & 12,47 & $(20568 ; 23608)$ \\
\hline 12 & 9466 & 1097 & 11,59 & $(7946 ; 10986)$ & 32 & 13334 & 2736 & 20,52 & $(11814 ; 14854)$ \\
\hline 13 & 8354 & 1177 & 14,09 & $(6834 ; 9874)$ & 33 & 13404 & 3082 & 23,00 & $(11884 ; 14924)$ \\
\hline 14 & 10394 & 1332 & 12,81 & $(8874 ; 11914)$ & 34 & 8783 & 2620 & 29,84 & $(7263 ; 10303)$ \\
\hline 15 & 22706 & 2362 & 10,40 & $(21118 ; 24293)$ & 35 & 23250 & 3343 & 14,38 & $(21730 ; 24770)$ \\
\hline 16 & 12845 & 2193 & 17,07 & $(11325 ; 14364)$ & 36 & 13741 & 2253 & 16,39 & $(12221 ; 15261)$ \\
\hline 17 & 18238 & 3781 & 20,73 & $(16718 ; 19758)$ & 37 & 20916 & 2328 & 11,13 & $(19396 ; 22436)$ \\
\hline 18 & 17716 & 2610 & 14,73 & $(16196 ; 19236)$ & 38 & 19901 & 3051 & 15,33 & $(18381 ; 21421)$ \\
\hline 19 & 18717 & 2728 & 14,58 & $(17197 ; 20237)$ & 39 & 18571 & 3244 & 17,47 & $(17051 ; 20091)$ \\
\hline 20 & 15302 & 2429 & 15,88 & $(13782 ; 16821)$ & 40 & 9880 & 1359 & 13,76 & $(8360 ; 11400)$ \\
\hline
\end{tabular}

Nota: $E_{\mathrm{c} 0}$ - rigidez na compressão; DP - desvio padrão; CV\% - coeficiente de variação; IC - intervalo de confiança. 
Tabela 6 - Resultados estatísticos obtidos da rigidez na tração, $\mathbf{E}_{\mathbf{t} 0}$ (MPa)

\begin{tabular}{c|c|c|c|c|c|c|c|c|c}
\hline ID & $\mathbf{E}_{\mathbf{t} \mathbf{0}}$ & $\mathbf{D P}$ & $\mathbf{C V} \%$ & $\mathbf{I C}$ & $\mathbf{I D}$ & $\mathbf{E}_{\mathbf{t} \mathbf{0}}$ & $\mathbf{D P}$ & $\mathbf{C V} \%$ & $\mathbf{I C}$ \\
\hline 01 & 20214 & 2784 & 13,77 & $(18327 ; 22100)$ & 21 & 14757 & 1906 & 12,89 & $(12959 ; 16555)$ \\
\hline 02 & 17024 & 2912 & 17,11 & $(15218 ; 18830)$ & 22 & 14370 & 2210 & 15,38 & $(12564 ; 16176)$ \\
\hline 03 & 14745 & 2370 & 16,07 & $(12939 ; 16551)$ & 23 & 17630 & 1705 & 9,67 & $(15824 ; 19436)$ \\
\hline 04 & 16040 & 2791 & 17,40 & $(14234 ; 17846)$ & 24 & 22148 & 1149 & 5,19 & $(20341 ; 23954)$ \\
\hline 05 & 14439 & 2161 & 14,97 & $(12633 ; 16245)$ & 25 & 14117 & 1815 & 12,85 & $(12311 ; 15923)$ \\
\hline 06 & 13212 & 2665 & 20,17 & $(11405 ; 15018)$ & 26 & 13608 & 2233 & 16,41 & $(11801 ; 15414)$ \\
\hline 07 & 12764 & 1832 & 14,35 & $(10958 ; 14570)$ & 27 & 20933 & 2712 & 12,96 & $(19127 ; 22739)$ \\
\hline 08 & 14087 & 2452 & 17,40 & $(12281 ; 15893)$ & 28 & 18590 & 1749 & 9,41 & $(16784 ; 20396)$ \\
\hline 09 & 12501 & 1097 & 8,77 & $(10695 ; 14307)$ & 29 & 14675 & 1410 & 9,61 & $(14494 ; 14856)$ \\
\hline 10 & 12920 & 1900 & 14,71 & $(11114 ; 14726)$ & 30 & 18184 & 4210 & 23,15 & $(16297 ; 20070)$ \\
\hline 11 & 15014 & 1722 & 11,47 & $(13991 ; 16037)$ & 31 & 19308 & 2392 & 12,39 & $(17502 ; 21114)$ \\
\hline 12 & 9920 & 1290 & 13,01 & $(8114 ; 11726)$ & 32 & 13548 & 1446 & 10,67 & $(11742 ; 15354)$ \\
\hline 13 & 9523 & 1924 & 20,21 & $(7717 ; 11329)$ & 33 & 13453 & 2082 & 15,48 & $(11647 ; 15260)$ \\
\hline 14 & 11051 & 1722 & 15,58 & $(9245 ; 12857)$ & 34 & 9172 & 1443 & 15,74 & $(7366 ; 10978)$ \\
\hline 15 & 21203 & 1504 & 7,09 & $(19397 ; 23009)$ & 35 & 21088 & 3532 & 16,75 & $(19202 ; 22974)$ \\
\hline 16 & 13382 & 1454 & 10,86 & $(11576 ; 15188)$ & 36 & 13859 & 2982 & 21,51 & $(12052 ; 15665)$ \\
\hline 17 & 16802 & 3195 & 19,02 & $(14996 ; 18608)$ & 37 & 21473 & 2773 & 12,91 & $(19667 ; 23279)$ \\
\hline 18 & 16698 & 2474 & 14,82 & $(14891 ; 18504)$ & 38 & 19476 & 4577 & 23,50 & $(17670 ; 21282)$ \\
\hline 19 & 18267 & 3105 & 17,00 & $(16461 ; 20073)$ & 39 & 17319 & 2087 & 12,05 & $(15513 ; 19125)$ \\
\hline 20 & 16148 & 2194 & 13,59 & $(14261 ; 18034)$ & 40 & 10442 & 1840 & 17,62 & $(8635 ; 12248)$ \\
\hline
\end{tabular}

Nota: $E_{\mathrm{t} 0}$ - rigidez na tração; DP - desvio padrão; CV\% - coeficiente de variação; IC - intervalo de confiança.

Primeiramente, ao se comparar tais propriedades com as espécies presentes na NBR 7190 (ABNT, 1997, p. 90, Anexo E), que traz um total de 43 espécies do grupo das folhosas, das quais 19 espécies são aqui avaliadas, verifica-se que todas estão dentro dos ICs aqui obtidos, sendo os valores médios, em sua maioria, próximos aos encontrados neste trabalho.

Destaca-se ainda o estudo realizado por Dias e Lahr (2004), que avaliaram 40 espécies de madeiras, dentre as quais 32 espécies são aqui avaliadas. Ao se comparar os valores médios para as propriedades mecânicas aqui estudadas, todas estão compreendidas dentro do IC, encontrando-se também em sua maioria próximas aos valores médios aqui obtidos.

É possível ainda comparar alguns valores individuais para algumas das espécies estudadas neste trabalho. Os valores médios aqui encontrados para a $f_{\mathrm{c} 0}$ se encontram dentro do IC e podem ser equiparados aos obtidos por Christoforo et al. (2017) para a madeira castelo $\left(f_{\mathrm{c} 0}=55 \mathrm{MPa}\right)$, Lahr et al. (2016a) para cambará-rosa $\left(f_{\mathrm{c} 0}=34 \mathrm{MPa}\right)$, Aquino et al. (2018) para copaíba $\left(f_{\mathrm{c} 0}=50 \mathrm{MPa}\right)$ e Tenorio e Moya (2018) para três idades de cedro-amargo, com o valor médio $f_{\mathrm{t} 0}=41,84 \mathrm{MPa}$.

Para a resistência $f_{\text {to }}$ Tenorio e Moya (2018) também pode ser citado ao apontar um valor médio $f_{\text {to }}=$ 40,11 MPa para o cedro-amargo, sendo este pouco abaixo do IC deste trabalho.

Para os módulos $\mathrm{E}_{\mathrm{c} 0}$ e $\mathrm{E}_{\mathrm{t} 0}$ podem-se citar os valores médios, obtidos por Lahr et al. (2016b), para a espécie jatobá extraída de três locais distintos, com os valores médios das três populações $\mathrm{E}_{\mathrm{c} 0}=21.881 \mathrm{MPa}$ e $\mathrm{E}_{\mathrm{t} 0}=21.583 \mathrm{MPa}$, sendo esses levemente abaixo dos valores médios, mas dentro dos ICs obtidos neste trabalho.

Com os valores médios, portanto, podem-se calcular os valores característicos (Equação 5), para as resistências na compressão e tração das 40 espécies de madeira avaliadas, juntamente com as respectivas classes de resistência (CR) (Tabela 7).

Ao considerar os 40 resultados para $f_{\text {c0,k }}$ constantes na Tabela 7 e os 40 resultados estimados pela Equação 7 $\left(f_{\mathrm{c} 0, \mathrm{k}}^{\text {Est }}=0,77 \cdot f_{\mathrm{t} 0, \mathrm{k}}\right)$, sendo os valores estimados associados aos valores característicos da resistência à tração, tais grupos $\left(f_{\mathrm{c} 0, \mathrm{k}}^{\text {Est }}\right.$ - resistência à compressão estimada pela Eq. 7$)$ e $\left(f_{\mathrm{c} 0, \mathrm{k}}\right.$ - resistência à compressão obtida experimentalmente) foram avaliados pela ANOVA, com resultados apresentados na Tabela 8. 
Da Tabela 8, constata-se a equivalência da média dos dois conjuntos de valores confrotados, o que revela a boa precisão da Equação 7 proposta pela normativa brasileira, cabendo destacar o atendimento $(p>0,05)$ da premissa de normalidade (Figura 1), o que valida os resultados obtidos da ANOVA.

Da relação $E_{c 0}=E_{t 0}$ (Equação 8), com base nos valores médios contantes nas Tabelas 5 e 6, os resultados da ANOVA para a análise da equivalência dos dois grupos de valores ( $E_{\mathrm{c} 0}$ - rigidez na compressão) e $\left(\mathrm{E}_{\mathrm{t} 0}-\right.$ rigidez na tração) são apresentados na Tabela 9 com os resultados do teste de normalidade de AndersonDarling apresentados conforme a Figura 2.

Tabela 7 - Resultados das resistências características (MPa) e classes de resistência (CR)

\begin{tabular}{l|l|l|l|l|l|l|c}
\hline \multicolumn{1}{c|}{ Espécies } & $\mathbf{C R}$ & $\boldsymbol{f}_{\mathbf{c 0 , \mathbf { k }}}$ & $\boldsymbol{f}_{\text {t0,k }}$ & \multicolumn{1}{c|}{ Espécies } & CR & $\boldsymbol{f}_{\mathbf{c 0 , k}}$ & $\boldsymbol{f}_{\text {t0,k }}$ \\
\hline Angelim-saia & C40 & 51,06 & 67,12 & Guajará & C60 & 72,37 & 83,43 \\
\hline Angelim-vermelho & C60 & 72,73 & 72,02 & Guarucaia & C40 & 53,30 & 63,98 \\
\hline Angico-branco & C40 & 41,87 & 53,94 & Itaúba & C60 & 61,70 & 72,60 \\
\hline Angico-preto & C40 & 55,55 & 76,65 & Jatobá & C60 & 79,60 & 125,29 \\
\hline Branquilho & C40 & 45,58 & 67,20 & Louro-preto & C40 & 42,70 & 61,36 \\
\hline Cafearana & C40 & 40,50 & 56,30 & Louro-verde & C40 & 45,07 & 69,87 \\
\hline Cambará-rosa & C20 & 27,30 & 31,79 & Maçaranduba & C60 & 73,70 & 109,40 \\
\hline Canafístula & C30 & 36,37 & 59,44 & Mandioqueira & C60 & 53,70 & 65,13 \\
\hline Castanheira & C30 & 38,93 & 61,95 & Oiticica-amarela & C60 & 58,00 & 74,23 \\
\hline Castelo & C40 & 54,54 & 86,42 & Oiuchu & C60 & 65,00 & 85,29 \\
\hline Catanudo & C40 & 50,91 & 48,79 & Parinari & C40 & 47,30 & 79,35 \\
\hline Cedro-amargo & C30 & 33,18 & 44,04 & Pau-óleo & C40 & 39,80 & 51,14 \\
\hline Cedro-doce & C20 & 29,99 & 48,55 & Piolho & C20 & 27,20 & 40,64 \\
\hline Cedroarana & C20 & 29,06 & 50,07 & Quarubarana & C60 & 55,00 & 81,65 \\
\hline Champanhe & C60 & 96,16 & 87,30 & Quina-rosa & C40 & 43,80 & 51,39 \\
\hline Copaíba & C40 & 44,13 & 52,67 & Rabo-de-arraia & C60 & 93,02 & 98,00 \\
\hline Cutiúba & C40 & 55,28 & 75,22 & Sucupira & C60 & 72,90 & 74,73 \\
\hline Garapa & C60 & 65,36 & 81,62 & Tachi & C40 & 45,20 & 65,05 \\
\hline Goiabão & C40 & 43,10 & 83,54 & Tatajuba & 46,60 & 39,99 \\
\hline Guaiçara & C40 & 58,92 & 80,59 & Umirana & C40 & &
\end{tabular}

Nota: $f_{\mathrm{c} 0, \mathrm{k}}$ e $f_{\mathrm{t} 0 \mathrm{k}, \mathrm{k}}$ - resistências característica à compressão e tração paralela às fibras.

Tabela 8 - Resultados da ANOVA para o conjunto de valores característicos da $\boldsymbol{f}_{\mathbf{c 0 , k}}^{\text {Est }}$ e $\boldsymbol{f}_{\mathbf{c o , k}}$

\begin{tabular}{l|c|c|c|c|c}
\multicolumn{1}{c|}{ Fonte } & GL & $\mathbf{S Q}_{\mathbf{a j}}$ & $\mathbf{Q M}_{\mathbf{a j}}$ & Valor $\mathbf{F}$ & P-valor \\
\hline Condição: $\left(f_{\mathrm{c} 0, \mathrm{k}}^{\text {Est }}=0,77 \cdot f_{\mathrm{t} 0, \mathrm{k}}\right)$ & 1 & 158,70 & 158,70 & 0,59 & 0,443 \\
\hline Erro & 78 & 20807,80 & 266,80 & -- & -- \\
\hline Total & 79 & 20966,50 & -- & -- & -- \\
\hline
\end{tabular}

Nota: GL - graus de liberdade; $\mathrm{SQ}_{\mathrm{aj}}$ - soma de quadrados; $\mathrm{QM}_{\mathrm{aj}}$ - quadrados médios. 
Figura 1 - Resultado do teste de Anderson-Darling para a resistência característica

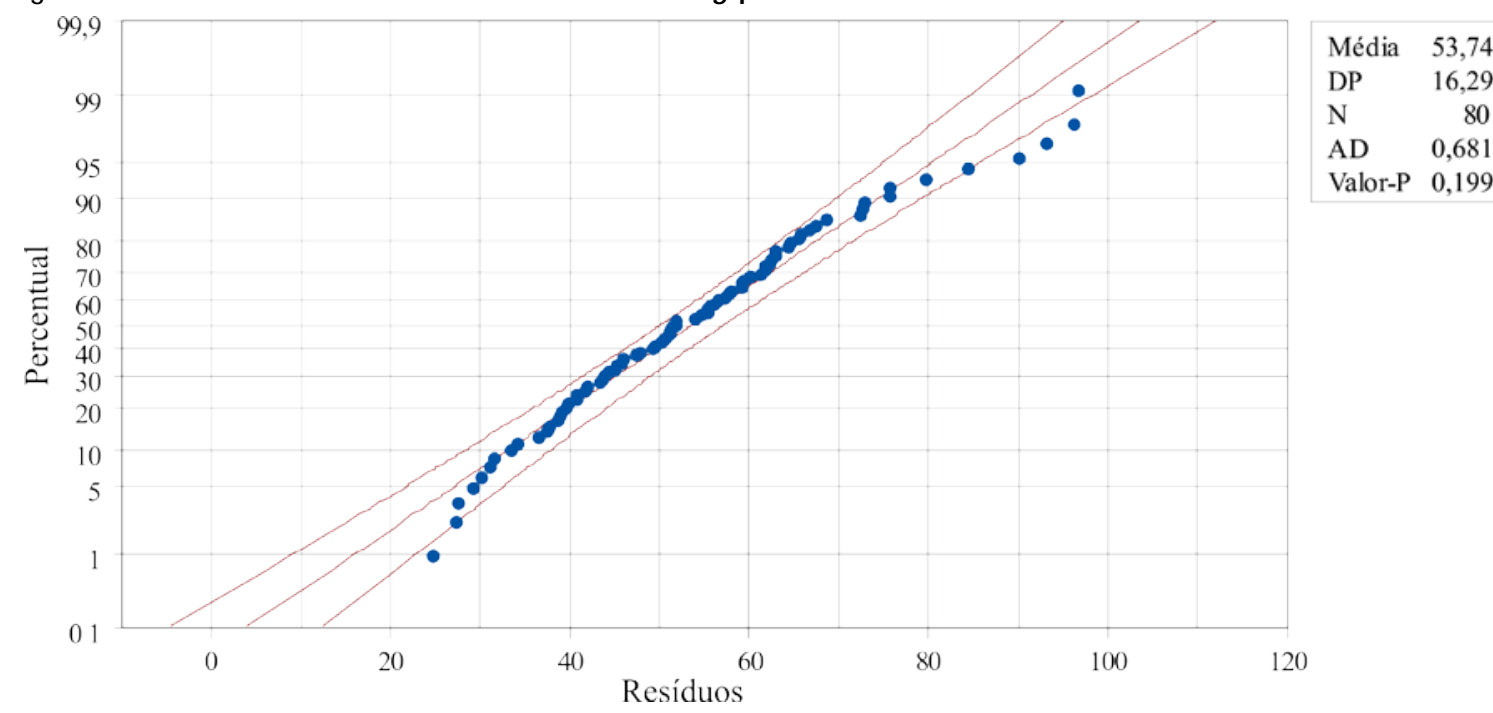

Tabela 9 - Resultados da ANOVA para o grupo de valores médios entre $\mathbf{E}_{\mathbf{c 0}}$ e $\mathbf{E}_{\mathbf{t} 0}$

\begin{tabular}{l|c|c|c|c|c}
\hline \multicolumn{1}{c|}{ Fonte } & GL & $\mathbf{S Q}_{\mathbf{a j}}$ & $\mathbf{Q M}_{\mathbf{a j}}$ & Valor $\mathbf{F}$ & P-valor \\
\hline Condição: $\left(\mathrm{E}_{\mathrm{c} 0}=\mathrm{E}_{\mathrm{t} 0}\right)$ & 1 & 1323294 & 1323294 & 0,09 & 0,768 \\
\hline Erro & 78 & 1180474968 & 1513429 & -- & -- \\
\hline Total & 79 & 1181798262 & -- & -- & -- \\
\hline
\end{tabular}

Nota: GL - graus de liberdade; $\mathrm{SQ}_{\mathrm{aj}}$ - soma de quadrados; $\mathrm{QM}_{\mathrm{aj}}$ - quadrados médios.

\section{Figura 2 - Resultado do teste de normalidade de Anderson-Darling para os valores de rigidez}

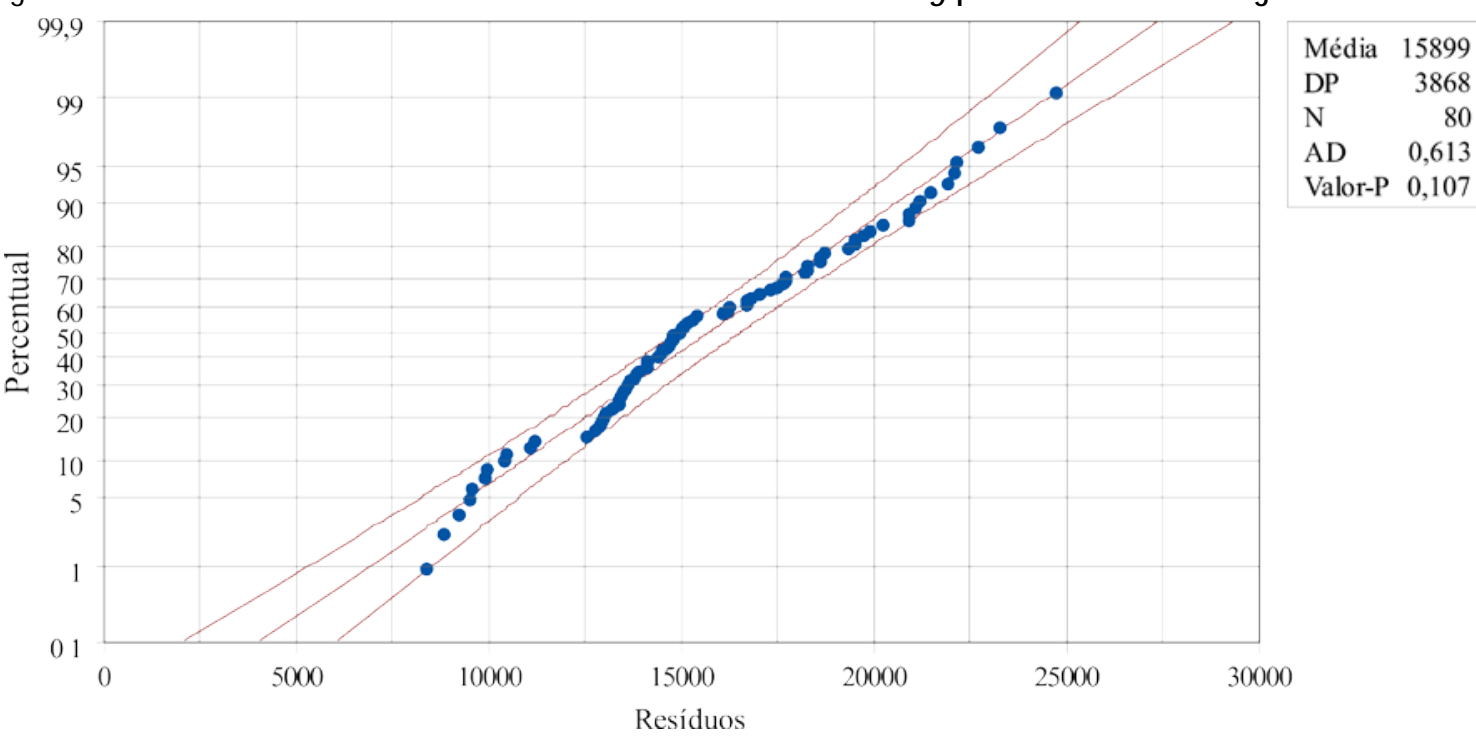

Pelo valor-p do teste de normalidade ser superior a significância adotada, constata-se a normalidade dos resíduos da ANOVA entre os valores de rigidez (Figura 2). Os resultados da Tabela 9 revelam a boa precisao da Equação $\mathrm{E}_{\mathrm{c} 0}=\mathrm{E}_{\mathrm{t} 0}$ proposta pela norma brasileira, assim como também ocorreu com a relação entre os valores característicos de resistência.

A partir do resultado médio da resistência característica à compressão para as 40 espécies de madeira, podese obter valores de $f_{\mathrm{c} 0, \mathrm{k}}^{\text {Est }} \approx 52 \mathrm{MPa}$, enquanto $f_{\mathrm{c} 0, \mathrm{k}} \approx 55 \mathrm{MPa}$ (Figura 3a). Para os módulos de elasticidade,

272 Wolenski, A. R. V.; Dias, F. M.; Peixoto, R. G.; Christoforo, A. L.; Lahr, F. A. R. 
como esperado, as médias para o $\mathrm{E}_{\mathrm{c} 0}$ e $\mathrm{E}_{\mathrm{t} 0}$ são estatisticamente equivalentes com valores $\approx 16.000 \mathrm{MPa}$ (Figura 3b).

Embora tenha sido constatada a validade das Equações 7 e 8, na sequência são apresentados os modelos de regressão, com base na análise de variância ( $F ; p<0,05)$, a fim de propor relações alternativas às equações existentes na normativa brasileira. Nas Tabelas 10 e 11 constam os resultados dos modelos obtidos para a resistência e rigidez, respectivamente.

Das Tabelas 10 e 11, todos os modelos foram considerados significativos $(\mathrm{F}, \mathrm{p}<0,05)$ pela ANOVA. As Figuras de 4 a 7 apresentam, respectivamente, os ajustes linear, exponencial, logarítmico e geométrico, visando estimar as resistências $f_{\mathrm{c} 0, \mathrm{k}}$ em função da $f_{\mathrm{t} 0, \mathrm{k}}$ e os módulos $\mathrm{E}_{\mathrm{c} 0}$ em função do $\mathrm{E}_{\mathrm{t} 0}$.

O modelo de melhor ajuste, eleito pelo maior coeficiente de determinação $\left(R_{a j}^{2}=65,11 \%\right.$ e $\left.R_{a j}^{2}=94,68 \%\right)$, foi o geométrico para ambas as relações avaliadas. Em adição, nota-se que todos os modelos evidenciam que o aumento nos valores característicos da $f_{\mathrm{c} 0, \mathrm{k}}$ ou do módulo $\mathrm{E}_{\mathrm{c} 0}$ implicam aumentos nos valores da $f_{\mathrm{t} 0, \mathrm{k}}$ e do $\mathrm{E}_{\mathrm{t} 0}$, como era de esperar. Em contrapartida, valores nulos para $f_{\mathrm{t} 0, \mathrm{k}}$ e $\mathrm{E}_{\mathrm{t} 0}$ só são nulos para a $f_{\mathrm{c} 0, \mathrm{k}}$ e $\mathrm{E}_{\mathrm{c} 0}$ para o modelo geométrico, o que reforça esse modelo como sendo o de melhor representatividade na estimativa dessas propriedades mecânicas.

Figura 3 - Valores médios e intervalos de confiança $(\mathbf{p}<0,05)$ para os grupos de valores da resistência característica à compressão $f_{\mathrm{c} 0, \mathrm{k}}^{\text {Est }}$ e $\boldsymbol{f}_{\mathrm{c} 0, \mathrm{k}}$ (a) e dos módulos de elasticidade $\mathrm{E}_{\mathrm{c} 0}$ e $\mathrm{E}_{\mathrm{t} 0}$ (b)

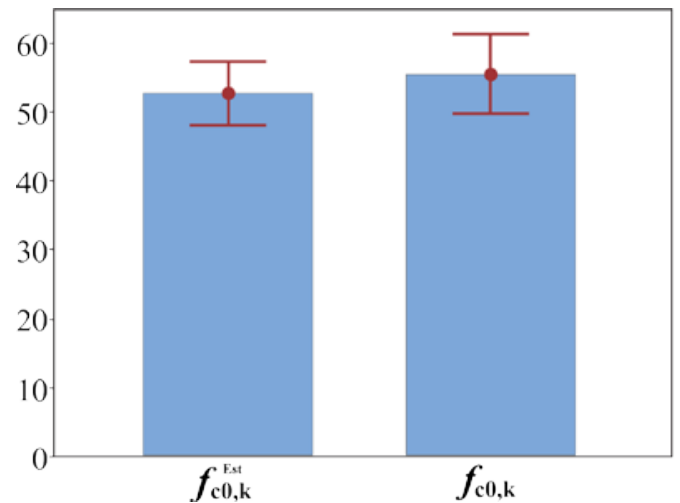

(a)

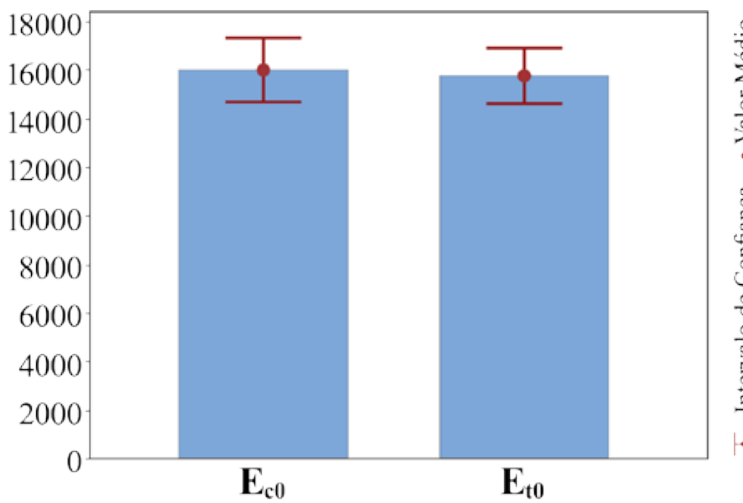

(b)

Tabela 10 - Resultados dos modelos de regressão para estimativa da resistência característica à compressão $\left(f_{\mathrm{c} 0, \mathrm{k}}\right)$ em função da resistência característica à tração $\left(f_{\mathrm{t} 0, \mathrm{k}}\right)$

\begin{tabular}{|c|c|c|c|c|c|}
\hline \multirow{2}{*}{ Modelos } & \multirow{2}{*}{ Equação } & \multicolumn{2}{|c|}{ Parâmetros } & \multirow{2}{*}{$\mathbf{P}_{\text {valor }}$} & \multirow{2}{*}{$\mathbf{R}_{\mathrm{aj}}^{2}$} \\
\hline & & $\mathbf{a}$ & b & & \\
\hline Linear & $f_{\mathrm{c} 0, \mathrm{k}}=\mathrm{a}+\mathrm{b} \cdot\left(f_{\mathrm{t} 0, \mathrm{k}}\right)$ & 3,85 & 0,75 & 0,000 & $62,42 \%$ \\
\hline Exponencial & $f_{\mathrm{c} 0, \mathrm{k}}=\mathrm{a} \cdot \mathrm{e}^{\mathrm{b} \cdot\left(f_{\mathrm{t} 0, \mathrm{k}}\right)}$ & 20,34 & 0,01 & 0,000 & $62,74 \%$ \\
\hline Logarítmico & $f_{\mathrm{c} 0, \mathrm{k}}=\mathrm{a}+\mathrm{b} \cdot \ln \left(f_{\mathrm{t} 0, \mathrm{k}}\right)$ & $-153,86$ & 49,99 & 0,000 & $61,43 \%$ \\
\hline Geométrico & $f_{\mathrm{c} 0, \mathrm{k}}=\mathrm{a} \cdot f_{\mathrm{t} 0, \mathrm{k}}^{\mathrm{b}}$ & 1,00 & 0,95 & 0,000 & $65,11 \%$ \\
\hline
\end{tabular}

Nota: a e b - parâmetros numéricos dos modelos de regressão; $R_{a j}^{2}$ - coeficiente de determinação.

Tabela 11 - Resultados dos modelos de regressão para a estimativa da rigidez na compressão $\left(E_{\mathbf{c} 0}\right)$ em função da rigidez na tração $\left(\mathrm{E}_{\mathrm{t} 0}\right)$

\begin{tabular}{c|c|c|c|c|c}
\hline \multirow{2}{*}{ Modelos } & \multirow{2}{*}{ Equação } & \multicolumn{2}{|c}{ Parâmetros } & \multirow{2}{*}{$\mathbf{P}_{\text {valor }}$} & \multirow{2}{*}{$\mathbf{R}_{\text {aj }}^{2}$} \\
\cline { 2 - 5 } & $\mathrm{E}_{\mathrm{c} 0}=\mathrm{a}+\mathrm{b} \cdot\left(\mathrm{E}_{\mathrm{t} 0}\right)$ & -1702 & 1,12 & 0,000 & $94,19 \%$ \\
\hline Linear & $\mathrm{E}_{\mathrm{c} 0}=\mathrm{a} \cdot \mathrm{e}^{\mathrm{b} \cdot\left(\mathrm{E}_{\mathrm{t} 0}\right)}$ & 5089 & 0,00007 & 0,000 & $92,47 \%$ \\
\hline Exponencial & $\mathrm{E}_{\mathrm{c} 0}=\mathrm{a}+\mathrm{b} \cdot \ln \left(\mathrm{E}_{\mathrm{t} 0}\right)$ & -147572 & 16972 & 0,000 & $92,75 \%$ \\
\hline Logarítmico & $\mathrm{E}_{\mathrm{c} 0}=\mathrm{a} \cdot \mathrm{E}_{\mathrm{t} 0} \mathrm{~b}$ & 0,31 & 1,12 & 0,000 & $94,68 \%$ \\
\hline Geométrico &
\end{tabular}

Nota: a e b - parâmetros numéricos dos modelos de regressão; $R_{a j}^{2}$ - coeficiente de determinação. 
Figura 4 - Regressão linear para estimativa da resistência à compressão $\left(f_{\mathrm{c} 0, \mathrm{k}}\right)$ em função da resistência à tração $\left(f_{\mathrm{t} 0, \mathrm{k}}\right)$ e da rigidez na compressão $\left(\mathrm{E}_{\mathrm{c} 0}\right)$ em função da rigidez na tração $\left(\mathrm{E}_{\mathrm{t} 0}\right)$
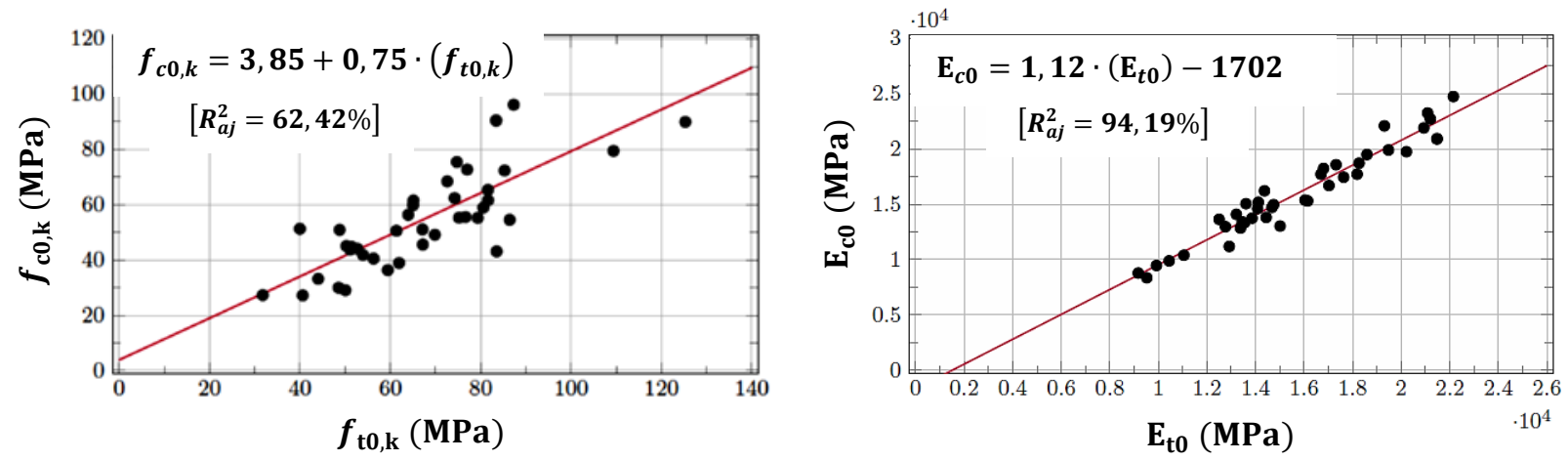

Figura 5 - Regressão exponencial para estimativa da resistência à compressão $\left(f_{\mathrm{c} 0, \mathrm{k}}\right)$ em função da resistência à tração $\left(f_{\mathrm{t} 0, \mathrm{k}}\right)$ e da rigidez na compressão $\left(\mathrm{E}_{\mathrm{c} 0}\right)$ em função da rigidez na tração $\left(\mathrm{E}_{\mathrm{t} 0}\right)$
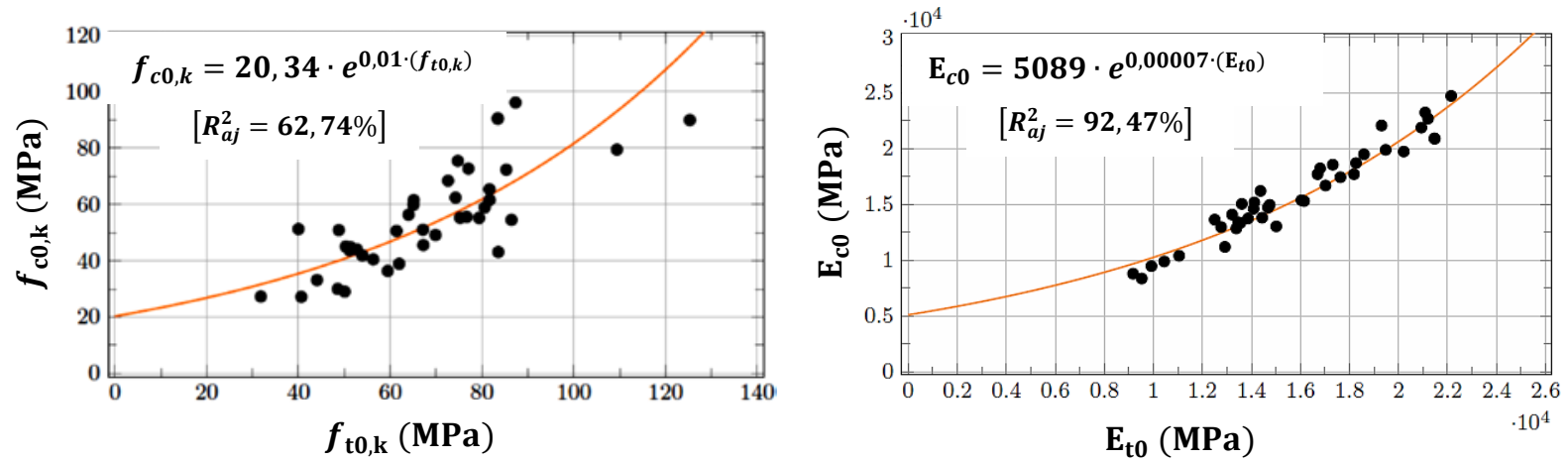

Figura 6 - Regressão logarítmica para estimativa da resistência à compressão $\left(f_{\mathrm{c} 0, \mathrm{k}}\right)$ em função da resistência à tração $\left(f_{\mathrm{t} 0, \mathrm{k}}\right)$ e da rigidez na compressão $\left(\mathrm{E}_{\mathrm{c} 0}\right)$ em função da rigidez na tração $\left(\mathrm{E}_{\mathrm{t} 0}\right)$
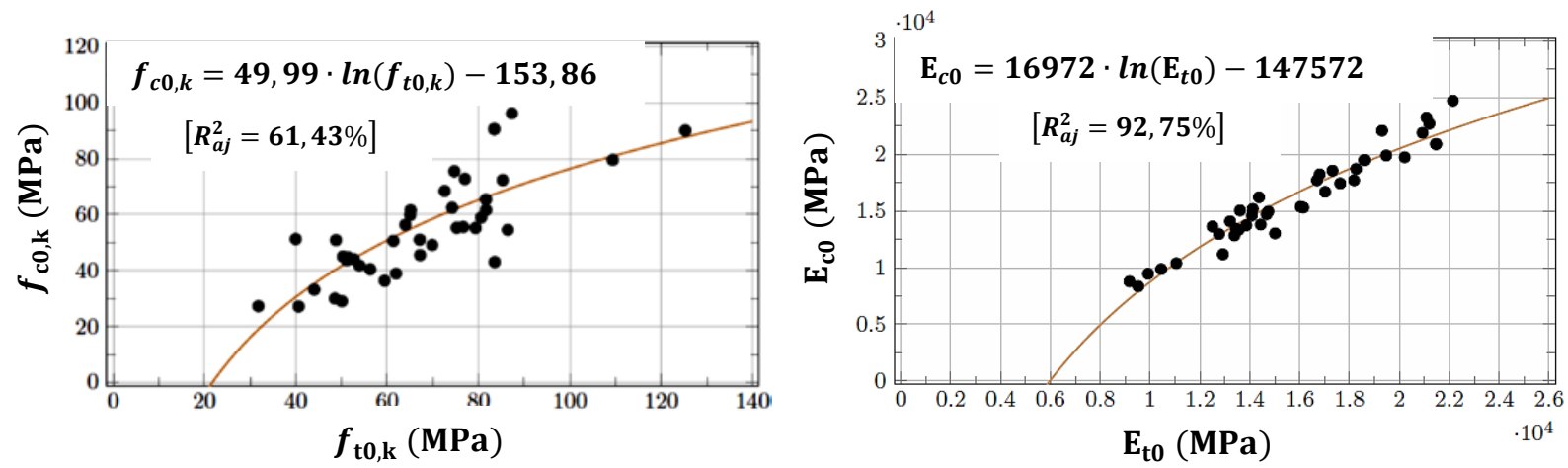

274 Wolenski, A. R. V.; Dias, F. M.; Peixoto, R. G.; Christoforo, A. L.; Lahr, F. A. R. 
Figura 7 - Regressão geométrica para estimativa da resistência à compressão $\left(f_{\mathrm{c} 0, \mathrm{k}}\right)$ em função da resistência à tração $\left(f_{\mathrm{t} 0, \mathrm{k}}\right)$ e da rigidez na compressão $\left(\mathrm{E}_{\mathrm{c} 0}\right)$ em função da rigidez na tração $\left(\mathrm{E}_{\mathrm{t} 0}\right)$
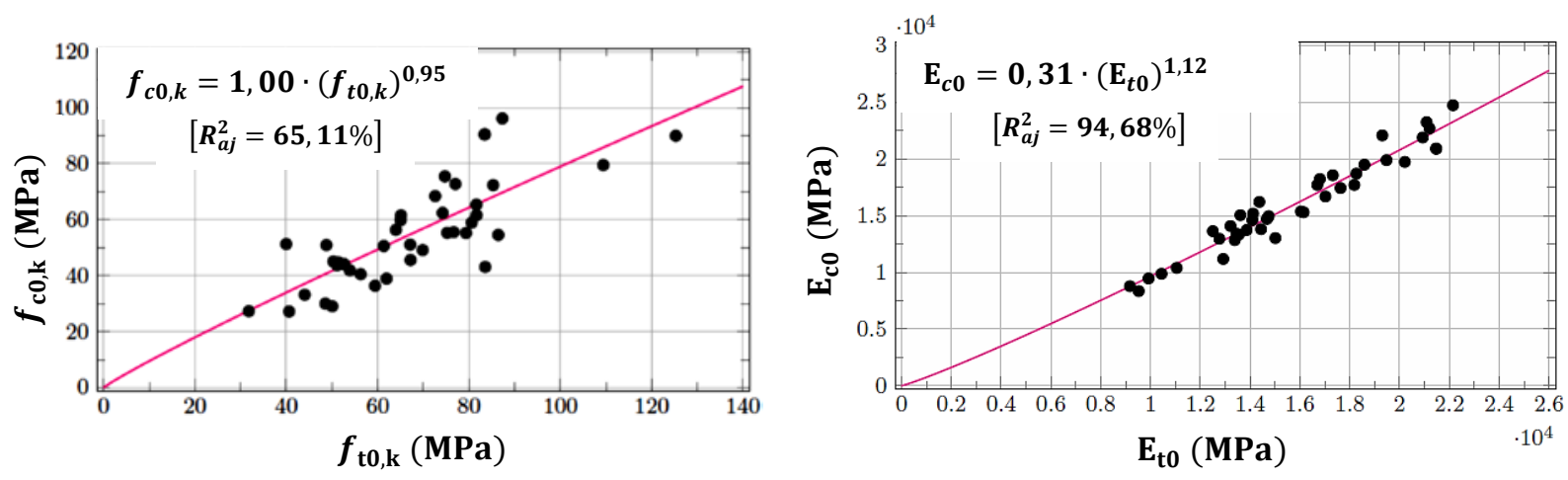

\section{Considerações finais}

Ao considerar as 40 espécies de madeira nativas usadas no presente estudo, as análises estatísticas permitem concluir que as relações $f_{\mathrm{c} 0, \mathrm{k}}=0,77 \cdot f_{\mathrm{t} 0, \mathrm{k}}$ e $\mathrm{E}_{\mathrm{c} 0}=\mathrm{E}_{\mathrm{t} 0}$ propostas pela NBR 7190 (ABNT, 1997) fornecem boa precisão e podem ser efetivamente adotadas na estimativa de tais propriedades, cabendo destacar que as expressões da referida norma levam em consideração, conjunta, sete espécies do grupo das coníferas e 43 do grupo das folhosas, fato esse preponderante e que justifica a realização deste trabalho.

Com relação aos modelos avaliados, a regressão geométrica apresentou o melhor ajuste na estimativa da resistência $\left(R_{a j}^{2}=65,11 \%\right)$ e rigidez $\left(R_{a j}^{2}=94,68 \%\right)$, conforme Figura 7 e Tabelas 10 e 11 . Tais coeficientes reforçam a aplicabilidade das funções propostas ao se apresentar como uma alternativa viável ao pré-dimensionamento de estruturas de madeiras folhosas, proporcionada pela ampla variabilidade de espécies avaliadas neste trabalho e pertencentes a todas as classes de resistência, segundo a normativa brasileira (ABNT, 1997, p. 16).

\section{Referências}

ALMEIDA, T. H. et al. Density as estimator of dimensional stability quantities of Brazilian tropical woods. BioResources, v. 12, n. 3, p. 6579-6590, 2017.

AQUINO, V. B. M. et al. Physical and mechanical characterization of Copaifera sp. wood specie. International Journal of Materials Engineering, v. 8, n. 3, p. 55-58, 2018.

ASSOCIAÇÃO BRASILEIRA DE NORMAS TÉCNICAS. NBR 7190: projeto de estruturas de madeira. Rio de Janeiro, 1997.

CAVALHEIRO, R. S. et al. Density as estimator of shrinkage for some Brazilian wood species. International Journal of Materials Engineering, v. 6, p. 107-112, 2016.

CHRISTOFORO, A. L. et al. Full characterization of Calycophyllum multiflorum wood specie. Journal of the Brazilian Association of Agricultural Engineering, v. 37, n. 4, p. 637-643, 2017.

SILVA, F. et al. Nondestructive evaluation of hardness in tropical wood. Journal of Tropical Forest Science, v. 26, n. 1, p. 69-74, 2014.

DADZIE, P. K.; AMOAH, M. Density, some anatomical properties and natural durability of stem and branch wood of two tropical hardwood species for ground applications. European Journal of Wood and Wood Products, v. 73, n. 6, p. 759-773, 2015.

DIAS, F. M.; LAHR, F. A. R. Estimativa de propriedades de resistência e rigidez da madeira através da densidade aparente. Scientia Forestalis, v. 65, p. 102-113, 2004.

FERNANDES, N. C. L. et al. Características físicas e anatômicas de Cedrela odorata L. e Cedrelinga cateniformis Ducke. Floresta e Ambiente, v. 25, n. 1, p. 1-10, 2018.

INTERNATIONAL ORGANIZATION FOR STANDARDDIZATION. ISO 13910: structural timber: characteristic values of strength-graded timber: sampling, full-size testing and evaluation. Genebra, 2005. 
JARDIM BOTÂNICO DO RIO DE JANEIRO. Flora do Brasil 2020 em construção. Disponível em: floradobrasil.jbrj.gov.br. Acesso em: 15 jul. 2019.

LAHR, F. A. R. et al. Avaliação de propriedades físicas e mecânicas de madeiras de Jatobá (Hymenaea stilbocarpa Hayne) com diferentes teores de umidade e extraídas de regiões distintas. Revista Árvore, v. 40, n. 1, p. 147-154, 2016b.

LAHR, F. A. R. et al. Full characterization of Erisma uncinatum warm. wood specie. International Journal of Materials Engineering, v. 6, n. 5, p. 147-150, $2016 \mathrm{a}$.

LOGSDON, N. B. et al. Avaliação dos estimadores da resistência característica à compressão paralela às fibras. Scientia Forestalis, v. 38, p. 579-587, 2010.

MATOS, G. S.; MOLINA, J. C. Resistência da madeira ao cisalhamento paralelo às fibras segundo as normas ABNT NBR 7190:1997 e ISO 13910:2005. Revista Matéria, v. 21, n. 4, p. 1069-1079, 2016.

RUELLE, J. et al. Variations in physical and mechanical properties between tension and opposite wood from three tropical rainforest species. Wood Science and Technology, v. 45, n. 2, p. 339-357, 2011.

SORIANO, J. et al. Wood density estimation using the sclerometric method. European Journal of Wood and Wood Products, v. 73, p. 753-758, 2015.

TENORIO, C.; MOYA, R. Evaluation of wood properties of four ages of Cedrela odorata trees growing in agroforestry systems with Theobroma cacao in Costa Rica. Agroforestry Systems, p. 1-16, 2018.

TIAGO, P. V. et al. Diversidade genética e estrutura populacional de Jatobá: uma espécie com potencial econômico para a Amazônia. Ciência Florestal, v. 28, n. 2, p. 515-524, 2018.

WEERAHANDI, S. ANOVA under unequal error variances. International Biometric Society, v. 51, p. 589-599, 1995.

\section{Agradecimentos}

Os autores agradecem o Conselho Nacional de Desenvolvimento Científico e Tecnológico (CNPq) pelo auxílio financeiro para o desenvolvimento deste trabalho.

\section{Anderson Renato Vobornik Wolenski}

Departamento de Engenharia Civil | Instituto Federal de Santa Catarina | Rua Aloísio Stoffel, 1271 | São Carlos - SC - Brasil | CEP 89885000 | Tel: (49) 3325-4149 | anderson. wolenski@ifsc. edu.br

\section{Fabrício Moura Dias}

Departamento de Engenharia Civil | Centro Universitário do Leste de Minas Gerais | Av. Tancredo Neves, 3500 | Coronel Fabriciano - MG Brasil | CEP 35170-056 | Tel: (31) 3846-5500 | E-mail: fmdias2@hotmail.com

\section{Rodrigo Guerra Peixoto}

Departamento de Engenharia de Estruturas | Universidade Federal de Minas Gerais | Av. Antônio Carlos, 6627| Belo Horizonte - MG Brasil | CEP 31270-901 | E-mail: rodrigo. peixoto@dees.ufmg.br

\section{André Luís Christoforo}

Departamento de Engenharia Civil | Universidade Federal de São Carlos | Rodovia Washington Luís, Km 235 | São Carlos - SP - Brasil | CEP 13565-905 | E-mail: christoforoal@yahoo.com.br

\section{Francisco Antonio Rocco Lahr}

Departamento de Engenharia de Estruturas | Universidade de São Paulo | Av. Trabalhador Sãocarlense, 400| São Carlos - SP - Brasil | CEP 13566-590 | Tel: (16) 3373-9455 | E-mail: frocco@sc.usp.br

\section{Ambiente Construído}

Revista da Associação Nacional de Tecnologia do Ambiente Construído

Av. Osvaldo Aranha, 99 - 30 andar, Centro

Porto Alegre - RS - Brasil

CEP $90035-190$

Telefone: +55 (51) 3308-4084

Fax: +55 (51) 3308-4054

www. seer. ufrgs. br/ ambienteconstruido

E-mail: ambienteconstruido@ufrgs.br 